\title{
Éducation à l'environnement en milieu scolaire et partenariat avec les collectivités territoriales - Une expérience en Auvergne
}

Christophe Andreux

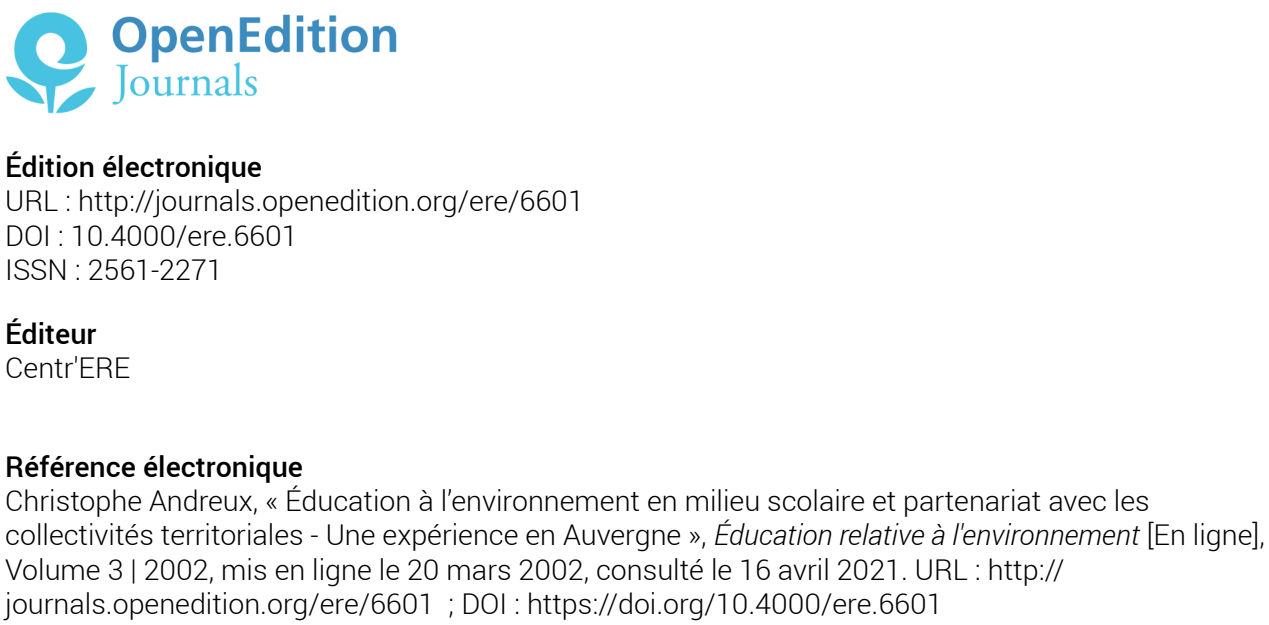

Ce document a été généré automatiquement le 16 avril 2021 


\title{
Éducation à l'environnement en milieu scolaire et partenariat avec les collectivités territoriales - Une expérience en Auvergne
}

\author{
Christophe Andreux
}

1 En Auvergne, vieille province du Massif central français, il est unanimement reconnu que le patrimoine naturel est exceptionnel; sa préservation et sa mise en valeur sont nécessaires pour faire de l'environnement un atout majeur de développement. Dans le cadre de leur projet de territoire, de plus en plus de collectivités prennent en compte l'environnement dans des dispositifs tels que les Chartes paysagères, environnementales, les Contrats de Rivières, les programmes de collecte sélective des déchets, etc. Pour accompagner ces politiques de territoire, la population et notamment les jeunes doivent être impliqués et responsabilisés, ce qui nécessite une prise de conscience de la valeur de leur environnement et de l'importance d'agir en sa faveur. C'est pourquoi des associations d'éducation à l'environnement telles que Espaces et Recherches, ainsi que le réseau régional d'éducation à l'environnement, le GRAINE Auvergne, sont régulièrement sollicitées par différents types de collectivités territoriales pour intervenir dans les écoles et les collèges essentiellement. Elles le font avec leurs propres méthodes et en particulier en mettant en œuvre une pédagogie de projet.

2 Pendant quatre ans, nous avons mené cette démarche d'accompagnement éducatif des politiques environnementales dans les écoles et les collèges. Du contact entre ces trois cultures, celle des associations, celle de l'école et celle des collectivités territoriales, est née une meilleure compréhension des buts de chacun et des limites encore existantes à ce type d'action. 


\section{Associations en milieu scolaire : pour des projets éducatifs communs}

\section{Collectivités et associations}

3 L'association Espaces et Recherches œuvre en Auvergne depuis 1978 pour protéger la nature, éduquer et former à l'environnement. Elle mène de multiples actions d'éducation à l'environnement (sensibilisation et animation) auprès de la population, en particulier en milieu rural, ainsi que de la formation des différents acteurs concernés par l'éducation à l'environnement (animateurs, éducateurs, enseignants, techniciens de collectivités et élus, etc.). Pour cela, elle compte dans son équipe cinq animateurs-formateurs spécialisés en environnement.

4 L'association GRAINE Auvergne, existe depuis 1994. C'est un groupement de professionnels composé d'individuels (éducateurs, animateurs, enseignants) et de structures ayant des compétences techniques et pédagogiques en éducation à l'environnement. Ces professionnels répondent à la demande de recherches et d'actions dans le domaine de l'éducation à l'environnement.

5 Ses objectifs sont :

- Mener des recherches en éducation à l'environnement suivies par des actions éducatives au niveau de la région Auvergne. Cette démarche de recherche est indissociable de l'action de terrain (animation, formation).

- Permettre l'accès à l'éducation à l'environnement pour tous les publics (pour éviter toute discrimination, notamment par l'argent).

- Développer et coordonner des recherches et des actions, et en effectuer le montage financier.

- Favoriser, initier et participer à une dynamique partenariale impliquant les différents acteurs régionaux (état, établissements publics et agences, collectivités territoriales, etc.) sur nos projets.

- Rassembler et mutualiser des forces et compétences dispersées sur le territoire régional.

6 Les collectivités territoriales ont pris conscience de l'utilité d'un travail de sensibilisation et d'éducation en environnement auprès des jeunes et notamment en milieu scolaire surtout après 1992. La responsabilité de l'application des lois de 1992 sur la gestion des déchets et de l'eau est donnée aux collectivités territoriales sur leur territoire. Ce contexte réglementaire a favorisé des initiatives pour l'élaboration et la mise en œuvre d'outils de planification et de gestion de leurs politiques environnementales (Charte, Contrat de Rivière, Plan de communication, etc.). Lors de leur mise en place, l'essentiel de ces programmes était centré sur les aspects techniques et réglementaires avec un petit volet « information de la population ». Très rapidement les collectivités territoriales ont identifié des problèmes de mise en œuvre, par la population, de ces nouveaux dispositifs de gestion. Une des raisons repose sur le fait que ces nouveaux dispositifs nécessitent des changements de comportement au quotidien et donc une prise de conscience du respect de leur environnement et de leur responsabilité en tant que citoyen. Ce constat a permis, au milieu des années 1990, une prise en compte plus importante de l'éducation à l'environnement.

7 Les programmes d'éducation à l'environnement mis en œuvre se sont centrés sur le public jeune et particulièrement à l'école et au collège pour trois raisons essentielles. La 
première est le rôle donné aux jeunes par les décideurs: ils constituent incontestablement des vecteurs auprès des adultes, de plus, ce sont les adultes de demain ! La seconde est liée au contexte éducatif et d'enseignement dans lequel se trouve le jeune ; il est plus pratique (et sans doute plus efficace) de travailler en groupeclasse, dans la durée, en s'intégrant et répondant de manière concrète aux grands thèmes des programmes scolaires, particulièrement des disciplines comme les Sciences de la Vie et de la Terre, l'Histoire et la Géographie ou l'Éducation civique. La troisième fait référence à des relations administratives et financières déjà existantes entre les écoles et les mairies et entre les conseils généraux (département) et les collèges; cellesci sont essentiellement basées sur la gestion des locaux et peuvent s'étendre, en fonction de la politique éducative de la collectivité, à des soutiens financiers pour des investissements et actions pédagogiques.

8 C'est ainsi que durant quatre ans, 21 programmes éducatifs ont été mis en œuvre pour six collectivités territoriales.

\section{Avec l'école et dans l'école}

9 La participation au projet est volontaire de la part des jeunes et des enseignants. S'ils s'engagent, chaque classe formulera un projet spécifique, tout en respectant la cohérence avec l'ensemble des autres initiatives liées à l'action globale. Il est impératif que la démarche engagée par la collectivité territoriale puisse s'inscrire dans les projets pédagogiques de circonscription, et par conséquent dans les projets d'établissement et/ ou de classe (A.E.I.).

10 Les collectivités territoriales sont donc porteuses de projets sur les aspects administratif, financier et de coordination générale et elles soumettent leurs intentions au Rectorat et à l'Inspection Académique pour favoriser un co-portage avec la prise en charge du volet éducatif et un suivi global des programmes. Il y a constitution d'un groupe de pilotage.

11 C'est pour cela que parmi les partenaires de l'opération figurent toujours de façon active le ministère de l'Éducation nationale qui intervient sur les plans financiers et techniques et l'Inspection Académique du département.

12 Une démarche d'évaluation et de valorisation des expériences et des productions sera engagée par les opérateurs (enseignants, animateurs et conseillers pédagogiques, etc.) et coordonnée par le groupe de pilotage (la collectivité, l'Éducation nationale et la structure d'éducation à l'environnement).

13 Afin d'optimiser sa mise en place, le programme est adapté à chaque cycle et à chaque niveau (du cycle 1 à la 3e). Pour les écoles maternelles et primaires, la répartition des interventions peut se dérouler sur une base modulable de 3 à 5 jours, sur 2 trimestres. Une adaptation est proposée aux classes de collèges pour que le temps d'intervention corresponde également aux contraintes structurelles.

\section{L'ouverture à d'autres collaborations}

14 Chaque programme a reçu l'appui et la collaboration d'un grand nombre de partenaires avec une spécificité pour chaque thème et territoire. Voici un exemple pour illustrer ce type de partenariat: celui du projet "HORIZONS» qui aborde la thématique des paysages pour la Communauté de Communes Sancy-Artense (63), en milieu rural 
montagnard. Durant l'année scolaire 1998/99, 150 enfants de 4-10 ans de 9 classes des communes de La Tour d'Auvergne, Bagnols, Saint-Sauves, Tauves, Saint-Donat et Avèze ont participé au projet mis en place sur 7 mois.

Parmi les partenaires, on peut distinguer :

- les partenaires financiers: l'Europe et son programme Leader Volcans II ; l'État, par les ministères de l'Éducation nationale, de l'Environnement, de la Jeunesse et des Sports, ou de l'Agriculture; le Conseil Régional, le Conseil Général du Puy-de-Dôme ainsi que d'autres organismes comme Électricité de France ou le Parc des Volcans d'Auvergne ;

- les partenaires techniques: en premier lieu, les élus, techniciens et habitants, des organismes comme l'Office National des Forêts, ou le CEMAGREF (Institut de recherche pour l'ingénierie de l'agriculture et de l'environnement), les Archives Départementales, le Parc des Volcans d'Auvergne, la Maison de l'Innovation, des grandes Écoles comme l'ENITA (École nationale d'ingénieurs des techniques agricoles) ;

- les partenaires médiatiques : journaux régionaux comme la Montagne, le Semeur, Hebdo Info Magazine, Magazine un, deux... quatre, la radio et la télévision régionales : France 3 Auvergne et Radio-France Puy-de-Dôme.

Le rôle de l'association, ici Espaces et Recherches, est spécifique. Elle est partenaire tout en jouant son rôle d'acteur de la société civile et missionnée comme expert par la collectivité territoriale. Ces missions recouvrent :

- les soutiens technique et méthodologique à la définition du programme et à l'élaboration de la dynamique partenariale ;

- les relations avec les partenaires techniques ;

- le recentrage du contenu pédagogique par rapport au projet global du territoire (Charte Paysagère) ;

- la participation à la coordination/animation générale du programme ;

- les soutiens méthodologiques et techniques auprès des classes (préparation, concertation, conception et mise à disposition d'outils et de documentation pédagogique et intervention dans les classes).

\section{Projets éducatifs : le territoire au cœur de la démarche}

\section{La démarche pédagogique}

17 Cette démarche intègre la pédagogie de projet et l'écoformation. Ces méthodes pédagogiques ont pour but :

- de développer et enrichir la relation directe entre le jeune et son environnement quotidien (relation sensorielle, affective, émotive, cognitive, etc.) ;

- d'amener le jeune à une meilleure perception de son milieu en développant ses sens, ses capacités d'observation, de compréhension, d'analyse et d'expression ;

- d'encourager le jeune à réfléchir, à proposer des solutions alternatives et à agir, à son niveau, dans le cadre des situations liées à l'environnement qu'il a rencontrées ;

- de conduire le jeune à une attitude écocitoyenne.

18 Sans entrer dans le détail, on peut prendre deux exemples menés dans des écoles primaires (à classe unique puisqu'il s'agit de petits villages de montagne). 


\section{La forêt dans le paysage d'Avèze}

\section{Plan de Recherche :}

- Comment s'appellent les arbres de la forêt?

- Qui a planté les premiers arbres?

- Pourquoi trouve-t-on des forêts avec des arbres tous identiques et alignés?

- Pourquoi a-t-on trouvé plusieurs sortes d'arbres au même endroit?

- D'où viennent tous ces arbres, comment sont-ils arrivés là?

- Pourquoi y a-t-il plus d'arbres à la campagne que dans les villes?

\section{Actions " agir et participer " :}

- Enquêtes, comparaison entre forêt régulière et forêt mixte, rencontre avec des bûcherons.

\section{Réalisations :}

- tableaux naturels représentant les arbres étudiés,

- fresque représentant la forêt dans leur paysage,

- panneaux explicatifs du vécu du projet par les enfants et de l'ensemble des résultats,

• jeu « quel arbre suis-je ? » avec feuilles, écorces et fruits ou graines.

\section{L'activité agricole dans le paysage de Saint-Donat}

\section{Plan de Recherche :}

- Pourquoi n'y a-t-il pas de blé à Saint-Donat?

- Comment sont faits les sols de Saint-Donat?

- Quelles sont les activités des agriculteurs de Saint-Donat aujourd'hui?

- Est-ce que le climat et la nature du sol ont une influence sur l'utilisation des terres?

-Avons-nous des indices d'une culture ancienne de blé ou d'autres céréales?

\section{Actions « agir et participer " :}

- $\circ$ Enquêtes auprès de la population locale (aubergistes, habitants du village, jardiniers locaux, parents, etc.).

\section{Réalisations :}

- $\circ$ Jardin potager

- Exposition : blé et seigle germés, dessins et textes, maquette du paysage de Saint-Donat et du Sancy.

\section{Trois grandes phases ponctuent la démarche pédagogique}

Les jeunes deviennent acteurs et responsables de leur apprentissage.

\section{Phase $1:$ Temps de représentations et d'éveil}

Exprimer ses représentations initiales et ses émotions, avoir un contact physique avec le paysage, imaginer et créer un paysage, restituer ses connaissances, accepter la subjectivité du regard de chacun. S'interroger face au paysage par des jeux de symbolisation permettant de poser des règles de communication et d'objectiver le 
regard. L'élève s'appropriera la thématique du paysage et sera dans une dynamique de découverte et d'interrogation.

\section{Phase 2 : Temps de définition du projet et de compréhension}

21 Se questionner, individuellement et collectivement, pour fixer ses attentes et envies de savoir. Émettre des hypothèses, définir un plan d'enquête et se donner les moyens d'atteindre ses objectifs. Les divers projets amèneront inévitablement l'enfant à s'interroger et tenter de comprendre les rôles et interactions des différents éléments composant son territoire d'étude. Ce sera l'occasion de s'initier à plusieurs «langages": les plans, cartes topographiques, enquêtes et rencontres auprès des différents acteurs, l'exploration du " raconté » (légendes, toponymie, etc.), la recherche sur toutes les composantes naturelles et environnementales du paysage. Ce temps de travail permettra d'utiliser de nombreux supports (bibliothèques, partenaires techniques, maquettes, expériences, multimédias, photographies, etc.), mais en conservant la notion d'alternance afin que l'enfant vise le double rapport de l'objectivité et de la subjectivité avec son paysage. Au terme de ces deux phases, l'élève aura construit un sens au paysage : il se le sera approprié et s'y sentira acteur.

\section{Phase 3 : Temps d'échanges, d'actions, de retransmission et de communication}

Partager les nouveaux savoirs, entre classes de différents niveaux du pays des Combrailles en favorisant l'échange entre primaire et collège, pour se construire une vision globale des territoires. Agir et participer : s'intégrer, par des actions concrètes, à la vie de son paysage. Transmettre ses acquisitions (spectacles, conférences, ouvrages, expositions, etc.) à un public choisi par la classe et réaliser une communication. À l'issue de cette phase, l'élève conciliera les notions d'évolution du paysage et de répercussions des activités humaines sur ce dernier. Libre à lui d'agir ensuite pour y placer sa pierre ou son arbre.

\section{Phase d'évaluation finale}

23 L'évaluation de l'ensemble du projet est réalisée par tous les acteurs: élèves, enseignants, animateurs et groupe de pilotage. La démarche de pédagogie de projet permet également aux enfants et à l'équipe d'encadrement une évaluation phase par phase.

Le projet de chaque classe prend vie durant les interventions ( 7 à 9 journées ou demijournées), réparties sur les deux premiers trimestres (octobre à avril), et dans un prolongement en classe et en travail personnel des élèves. Dans ce cadre, l'animateur(trice) suit la classe du début à la fin en apportant un soutien méthodologique (mise en œuvre des différentes phases, approches pédagogiques liées aux thèmes sur le terrain et en classe) et technique (connaissances naturalistes et environnementales).

Une démarche d'évaluation formative et de valorisation des expériences et des productions est engagée par les opérateurs (enseignants, animateurs, conseillers pédagogiques, etc.) et coordonnée par un comité de suivi. 


\section{La prise en compte du territoire}

L'environnement étudié est systématiquement celui de la commune où se trouve l'école. Les raisons sont nombreuses. Cela donne aux jeunes une meilleure connaissance de leur lieu de vie et de ses problèmes spécifiques et permet une appropriation de leur environnement. Ils en deviennent les acteurs d'aujourd'hui et de demain.

À partir de cette appropriation communale, nous avons cherché une mise en cohérence au niveau intercommunal de plusieurs projets sur le même thème parce qu'il nous semble indispensable qu'à partir de leur vie quotidienne, les jeunes puissent prendre conscience de l'identité de leur territoire, de leur "pays ", à travers ses spécificités paysagère, socio-économique, culturelle, administrative (par exemple : regroupement scolaire pour des petits effectifs). Ceci permet d'aborder une problématique environnementale, de manière pratique, globale et pertinente, à l'échelle d'une entité cohérente (bassin versant, unité paysagère). Et, au-delà, grâce à la notion d'interdépendance, d'approcher l'intercommunalité, qui se traduit par l'existence de la collectivité territoriale porteuse du projet éducatif.

Enfin, nous avons cherché, avec plus ou moins de succès, à ouvrir les projets aux différents acteurs locaux (élus, techniciens, administrations, spécialistes, habitants, etc.) pour leur permettre de participer activement au projet porté par les jeunes et de s'impliquer (concertation, partenariat, sensibilisation du reste de la population). Cela permet aux jeunes d'appréhender la complexité du fonctionnement d'une collectivité et la diversité, dont ils font partie, des acteurs qui peuvent y jouer un rôle.

\section{Difficultés et réussites d'une démarche partenariale}

\section{L'attitude des collectivités}

La mise en place de ces programmes éducatifs financés par les collectivités, et concernant plusieurs établissements scolaires d'un même territoire, met en lumière un certain nombre d'intérêts, de facteurs, influant sur l'attitude des collectivités.

La phase d'élaboration et de préparation du programme éducatif nécessite l'élaboration d'un partenariat financier, technique et médiatique. C'est notamment le «technique » et le "médiatique » qui vont valoriser les différents acteurs concernés par le territoire et le thème. Cela va permettre à la collectivité " porteuse de projets » de s'impliquer dans la coordination et sur le terrain en apportant ses compétences aux autres acteurs. Ceci, pour chacun des projets de classe, dès les premières phases, puis au cours d'échanges, d'actions, de retransmissions et communications.

31 C'est une démarche qui permet de fédérer de multiples acteurs autour des jeunes pour un projet commun. C'est aussi un moyen de créer de nouveaux liens entre collectivités, établissements scolaires et structures d'éducation à l'environnement, où chacun est indispensable pour que les projets de classe puissent prendre toute leur dimension éducative et communautaire. Lorsque l'on parle de collectivité, cela concerne les élus et les techniciens, mais également les habitants, les acteurs locaux, etc.

Une possibilité est donnée à la population, à travers les jeunes, de participer activement et de faire acte de civisme dans une démarche participative. Ce qui permet 
à la collectivité d'œuvrer pour une écocitoyenneté et de revaloriser son rôle auprès de ses administrés.

\section{Un exemple de démarche participative}

En 1997, sur le thème de l'eau, les élèves de la classe de 6e du collège de Saint-Sauves (Commune de Sancy-Artense), après avoir travaillé sur la qualité de la rivière qui traverse leur village, ont réalisé un petit document à destination de la population pour présenter le résultat de leurs recherches (mauvais indice biotique et qualité piscicole, décharges sauvages, etc.). Ils ont en parallèle demandé une rencontre avec le Conseil Municipal pour organiser, avec l'autorisation et le soutien technique et financier de la mairie, un nettoyage de la rivière. La rencontre s'est conclue par un accord sur la totalité de leur demande et lors de la mise en place du nettoyage, le document ayant rempli son objectif de sensibilisation, les élèves ont reçu l'aide de bon nombre d'habitants et de partenaires techniques sollicités durant les phases précédentes. Lors de cette même journée, un temps était prévu pour que la population puisse visiter une exposition conçue et animée par les élèves. Celle-ci présentait leur démarche d'investigation de terrain, les rencontres avec des partenaires techniques (garde pêche, entreprise gérant la station d'épuration et exploitants agricoles), les enquêtes auprès de leurs familles et des commerçants, les résultats de leurs recherches (plus détaillées que le document). Y figuraient également des poèmes, des fresques et des maquettes exprimant la désapprobation des élèves face à cette situation et les solutions envisagées.

\section{Des freins identifiés}

On fait souvent l'amalgame entre les programmes d'information, de sensibilisation, et d'éducation à l'environnement. Pourtant, les objectifs de chacun des programmes ne sont pas les mêmes et les moyens techniques, financiers et humains diffèrent en conséquence. L'acte éducatif mené par les associations est régulièrement mal compris et donc dévalorisé, quelquefois remis en cause par les collectivités et par l'Éducation nationale pour les programmes scolaires. Ceci est lié à des raisons différentes suivant les acteurs.

Pour les collectivités, le problème se situe souvent dans le rapport entre le budget et le nombre de personnes concernées directement par le programme éducatif. Ce rapport apparaît comme beaucoup moins intéressant que celui d'un programme d'information, la justification n'est pas toujours facile. Quelques raisons ressortent :

- le fait de confier le programme éducatif au service communication de la collectivité avec le risque de ne pas rencontrer un personnel sensibilisé ou compétent pour piloter un tel programme ;

- le manque de reconnaissance du professionnalisme des structures d'éducation à l'environnement, souvent des associations avec animateurs professionnels salariés, génère une incompréhension de leurs coûts d'intervention ;

- enfin, un problème d'image des associations d'éducation à l'environnement assimilées à des «écolos contestataires" par les collectivités. En effet, un grand nombre d'associations d'éducation à l'environnement ont été ou sont encore des associations de protection de la nature et de l'environnement. 
Pour l'Éducation nationale, les difficultés résident plus dans la compréhension et l'acceptation de la complémentarité entre l'éducation formelle et non formelle. Les considérations de sécurité et de sérieux éducatif des intervenants entre en jeu, mais aussi un effet "consommation d'actions éducatives" pour certains enseignants qui n'ont pas de réel projet intégrant les interventions. Depuis 1995/96, ces problèmes ont été sérieusement pris en compte par un certain nombre d'acteurs, mais le passé est encore bien prégnant et suppose un travail étroit entre tous.

\section{Rendre l'éducation à l'environnement crédible}

La démarche de pédagogie de projet et d'écoformation donne une validité sur le sérieux éducatif de la structure d'éducation à l'environnement. Désormais, les collectivités, l'Éducation nationale ainsi que les autres partenaires perçoivent mieux le rôle des structures d'éducation à l'environnement comme étant des médiateurs entre les différents acteurs (porteur de projet, partenaires, classes, etc.) et l'environnement naturel et culturel de leur territoire.

Enfin, c'est par la rencontre dans cet espace d'échange qu'est le projet, en «faisant ensemble ", que l'on identifie d'autres besoins d'actions qui peuvent prolonger le projet auprès des jeunes, mais aussi auprès des adultes.

\section{En conclusion}

Il ressort de ces expériences quelque chose de fort. La pédagogie de projet comme méthode d'éducation active auprès des jeunes peut tout à fait se transposer, s'adapter et s'articuler pour une mobilisation et une implication de l'ensemble des différents acteurs pour des projets de développement durable de territoires. Les collectivités territoriales peuvent effectivement avoir une implication partenariale qui dépasse le seul financement.

40 Aujourd'hui, au regard des difficultés et des limites identifiées, un chantier important nous attend pour que toutes les expériences similaires à l'échelle du territoire national, voire international, puissent bénéficier d'un véritable état des lieux avec évaluation, théorisation, mutualisation par un travail de recherche-action ou de recherchedéveloppement mené en étroit partenariat entre les différents acteurs.

41 Ceci contribuerait à donner à chacun des moyens de transfert méthodologique adaptables aux contextes toujours différents. Ceci permettrait également une reconnaissance de l'éducation à l'environnement et une légitimité au soutien réel qu'elle peut apporter à la mise en œuvre des politiques territoriales où les notions de solidarité, d'équité et de responsabilité sont de mise. 


\section{BIBLIOGRAPHIE}

Cottereau, D. (1997). Alterner pour apprendre. Montpellier : École et Nature.

Cottereau, D. (1994). À l'école des éléments. Lyon : Chroniques Sociales.

École et Nature. (2000). Programme pédagogique sur la gestion des déchets et la consommation «ROULETABOULE ». Cinquième édition. Montpellier : École et Nature.

École et Nature. (1999). Monter son projet, guide pratique d'éducation à l'environnement. Quatrième édition. Lyon : Chroniques Sociales.

École et Nature. (1997). Programme pédagogique sur l'eau « RICOCHETS». Montpellier : École et Nature.

École et Nature. (1996). Éduquer à l'environnement par la pédagogie de projet. Deuxième édition. Paris : L'Harmattan.

Espinassous, L. (1996). PISTES, pour la découverte de la nature et de l'environnement. Toulouse : Édition Milan.

\section{AUTEUR}

\section{CHRISTOPHE ANDREUX}

Christophe Andreux a reçu une formation professionnelle d'éducateur à l'environnement (BEATEP) et d'accompagnateur en montagne (BEES AMM). De 1996 à 2001, il a été responsable pédagogique, animateur et formateur pour l'association « Espaces et Recherches ", ainsi que coordinateur régional pour le réseau GRAINE Auvergne. De 1997 à 1998, il a aussi été chef de projet sur le programme éducatif national « Gestes au Quotidien » pour le réseau « École et Nature ». Il est aujourd'hui consultant et chargé de mission pour des porteurs de projets d'ERE avec un accent pour des territoires en milieu rural montagnard. Pour cela, il a suivi en parallèle un D.U.H.E.P.S sur 3 ans au SUFCO de l'Université F. Rablais de Tours. 